\title{
Generation of Superposition Spin States in an Atomic Ensemble
}

\author{
S. Massar * \\ Service de Physique Théorique, Université Libre de Bruxelles, \\ C.P. 225, Bvd. du Triomphe, 1050 Bruxelles, Belgium \\ E. S. Polzik \\ QUANTOP - Danish National Research Foundation Centre for Quantum Optics, \\ Niels Bohr Institute, Copenhagen, DK-2100, Denmark
}

(Dated: December 2, 2002)

\begin{abstract}
A method for generating a mesoscopic superposition state of the collective spin variable of a gas of atoms is proposed. The state consists of a superposition of the atomic spins pointing in two slightly different directions. It is obtained by using off resonant light to carry out Quantum Non Demolition Measurements of the spins. The relevant experimental conditions, which require very dense atomic samples, can be realized with presently available techniques. Long-lived atomic superposition states may become useful as an off-line resource for quantum computing with otherwise linear operations.
\end{abstract}

PACS numbers: 03.67.Mn; 42.50.Ct; 42.50.Dv

Generation of superpositions of macroscopically different quantum states have been attracting fundamental interest for a long time [1] since they illustrate the superposition principle in an extreme setting. Recently, such states have also been proposed as an off-line entanglement resource for a quantum computer, with which the computer can work using only linear operations [2]. Nondeterministically produced "cat" states of an ensemble of particles serve in this proposal as an alternative to operations with maximally entangled quantum states in other quantum computing schemes.

Superpositions involving a few microwave photons in a cavity have been demonstrated in [3]. Quantum superpositions have been recently also demonstrated using currents in superconducting interference devices [4]. There are proposals for realising such states in different physical systems, see for instance [5] for those which involve Bose Einstein Condensates (BEC). Methods for generating superpositions of two coherent states $|\alpha\rangle+|-\alpha\rangle$ of the electro-magnetic (e.m.) field have been suggested, but they require a perfect measurement of the photon number [6], which is impractical at present.

In this paper, we propose a method for realizing a superposition state of the collective spin variables of a gas of alkali atoms. Specifically the state that would be realised is a coherent superposition of the atomic spins pointing in two slightly different directions. Our method, in which the state of the atomic spins is generated via an interaction with light pulses, is another step within the growing field devoted to developing a quantum interface between light and atomic ensembles. Strong coupling between collective degrees of freedom of multi-atom ensembles and multi-photon pulses of light can be achieved in free space and has been recently used to demonstrate squeezing of

\footnotetext{
*Also at: Ecole Polytechnique, C.P. 165, Université Libre de Bruxelles, 1050 Brussels, Belgium
}

the collective spin [10,11] and entanglement between two atomic samples [9, 12]. This should be contrasted with the strong coupling of individual photons to individual atoms, which requires high finesse cavities [3, 7].

The method we propose here for generating a coherent superposition of the spins advances these techniques beyond the continuous variables approximation. Indeed in our proposal a strong atom-photon coupling has to be realized with a small number of atoms. The relevant experimental conditions, which require very dense atomic samples, seem to be realisable using presently available techniques. The transfer of the atomic ensemble spin state onto the state of light is also in principle possible [8, 13], and therefore superpositions of coherent states of light could also be realised using our method.

The collective spin variables for the ground state of an atomic ensemble $\hat{\vec{J}}=\sum_{i} \hat{\vec{J}}^{(i)}$, where $\hat{\vec{J}}^{(i)}$ is the total angular momentum of the $i$ th atom in the ground state, obey the usual commutation relations $\left[\hat{J}_{x}, \hat{J}_{y}\right]=i \hat{J}_{z}$. For simplicity we assume that each atom is a spin $1 / 2$ system. We start with the coherent spin state with maximal total angular momentum (i.e. all spins parallel) whereupon $\hat{J}_{x}^{2}+\hat{J}_{y}^{2}+\hat{J}_{z}^{2}=\frac{N^{a}}{2}\left(\frac{N^{a}}{2}+1\right)$ where $N_{a}$ is the number of atoms. We will suppose that the evolution of the collective spin is limited to unitary transfirmations without dissipation. Under these conditions the total length of the spin vector is conserved, i.e. it stays on the surface of the same Bloch sphere. Throughout the paper the spin vector stays polarized close to the $+z$ direction (close to $\left|\uparrow_{z}\right\rangle$ state), but not necessarily exactly in this direction. We can then define the rescaled variables $\hat{x}_{A}=\sqrt{\frac{2}{N_{a}}} \hat{J}_{x}$, $\hat{p}_{A}=\sqrt{\frac{2}{N_{a}}} \hat{J}_{y}, \hat{n}_{A}=\frac{N_{A}}{2}-\hat{J}_{z}$, which satisfy

$$
\begin{aligned}
{\left[\hat{x}_{A}, \hat{p}_{A}\right] } & =i+O\left(\hat{n}_{A} / N_{A}\right), \\
\frac{\hat{x}_{A}^{2}}{2}+\frac{\hat{p}_{A}^{2}}{2} & =\hat{n}_{A}+\frac{1}{2}+O\left(\hat{n}_{A}^{2} / N_{A}\right) .
\end{aligned}
$$

Thus $\hat{x}_{A}$ and $\hat{p}_{A}$ are analogous to the position and mo- 
mentum operators, or to the quadrature operators of a single mode of the e.m. field, and $\hat{n}_{A}$, the number of spins polarised in the $+z$ direction, is analogous to the occupation number of a harmonic oscillator, or to the number of photons in a mode of the e.m. field. The new feature of the present model, as compared to the earlier work, is that it goes beyond the harmonic oscillator approximation since the deviation of $\hat{J}_{z}$ from $N_{a} / 2$ is taken into account to first order.

The qualitative outline of the procedure for the "cat" state generation goes as follows, see figure 1 As shown in 8,9 , 11] a component of the collective spin operator can be measured in a QND way. A measurement of $\hat{J}_{x}$ to precision $\Delta J$ produces a squeezed state of the atomic spin with uncertainties $\Delta x_{A}^{2}=2 \Delta J^{2} / N_{a}=1 /\left(2 \xi^{2}\right)$ and $\Delta p_{A}^{2}=\xi^{2} / 2$ where $\xi$ denotes the degree of squeezing. Such a state has a "banana" shape localized around the pole of the Bloch sphere. The critical next step is to carry out a QND measurement of $\hat{J}_{z}$, i.e. of the number operator $\hat{n}_{A}$, with the same precision $\Delta J$. Such a measurement can be visualized as intersecting the "banana" with a plane parallel to the equatorial plane 1 The distance from the plane to the pole depends on the result of the QND measurement of $\hat{n}_{A}$. The "width" of the plane depends on the precision $\Delta J$ with which $\hat{n}_{A}$ is measured. The resulting state lies at the intersection of the plane and the initial squeezed state. It is a superposition state. Of central importance is that only "weak" QND measurements of the atomic population, i.e. measurements of $\hat{n}_{A}$ which do not need to have the single atom accuracy, are required to produce the superpostion state.

Let us now describe in detail the QND measurements of the atomic spin. The starting point is the effective interaction between the off resonant light and the atomic ground state spin [8, 11, 12]. Atomic levels and interactions with light are shown in figure 1a). Let us suppose that initially the photons are in the coherent state $\left|\psi_{P}\right\rangle=\int d x_{P} \exp \left[-x_{P}^{2} / 2\right]\left|x_{P}\right\rangle$ (hereafter we omit for simplicity normalisation constants) and that the atoms are polarised in the $z$ direction in the coherent spin state $|0\rangle_{n_{A}}=\int d x_{A} \exp \left[-x_{A}^{2} / 2\right]\left|x_{A}\right\rangle$ (the level scheme on top in figure 1a). For light propagating along the $x$ axis the effective interaction Hamiltonian is $H_{e f f}=\kappa \sum_{i}\left|\uparrow_{x}\right\rangle_{i}\left\langle\uparrow_{x}\left|\hat{a}_{L}^{\dagger} \hat{a}_{L}+\right| \downarrow_{x}\right\rangle_{i}\left\langle\downarrow_{x}\right| \hat{a}_{R}^{\dagger} \hat{a}_{R}$ where $\hat{a}_{L}, \hat{a}_{R}$ are the annihilation operators for left and right circular polarized light (the interaction is pictorially shown in the middle level scheme in figure 1a). We introduce the Stokes operators $\hat{S}_{x}=\hat{a}_{L}^{\dagger} \hat{a}_{L}-\hat{a}_{R}^{\dagger} \hat{a}_{R}$, $\hat{S}_{y}=\hat{a}_{L}^{\dagger} \hat{a}_{R}+\hat{a}_{R}^{\dagger} \hat{a}_{L}, \hat{S}_{z}=-i \hat{a}_{L}^{\dagger} \hat{a}_{R}+i \hat{a}_{R}^{\dagger} \hat{a}_{L}$ and assume that $\hat{S}_{z} \simeq N_{p}$ is close to its maximal value (where $N_{P}$ is the number of photons in the beam). Then we can define the rescaled position-momentum like operators $\hat{S}_{x}=\hat{x}_{P} \sqrt{N_{p} / 2}, \hat{S}_{y}=\hat{p}_{P} / \sqrt{N_{P} / 2}$ which obey $\left[\hat{x}_{P}, \hat{p}_{P}\right]=i$. With this notation the unitary evolution can be written (up to an unimportant overall phase) as

$$
U=\exp \left(-i a \hat{J}_{x} \hat{S}_{x}\right)=\exp \left(-i \alpha \hat{x}_{A} \hat{x}_{P}\right)
$$

where $\alpha=a \sqrt{N_{a} N_{p}} / 2$.

In the first step a spin squeezed state is obtained as follows 8, 11, 12] After the interaction with light the state is, in the $p_{P}$ basis, $U\left|\psi_{P}\right\rangle|0\rangle_{n_{A}}=\int d x_{A} d p_{P} \exp \left[-\left(\alpha x_{A}-\right.\right.$ $\left.\left.p_{P}\right)^{2} / 2\right] \exp \left[-x_{A}^{2} / 2\right]\left|x_{A}\right\rangle\left|p_{P}\right\rangle$. Upon measuring $\hat{p}_{P}$ a particular outcome $p_{P}$ is obtained. The state of the atoms conditioned on the measurement outcome $p_{P}$ is

$\psi_{A}\left(p_{P}\right)=\int d x_{A} \exp \left[-\left(\alpha x_{A}-p_{P}\right)^{2} / 2\right] \exp \left[-x_{A}^{2} / 2\right]\left|x_{A}\right\rangle$.

This is a squeezed state. By a rotation one can center the state around $p_{P}=0$ whereupon one has

$$
\begin{aligned}
\psi_{A}^{\text {squeezed }} & =d x_{A} \exp \left[-\left(\alpha^{2}+1\right) x_{A}^{2} / 2\right]\left|x_{A}\right\rangle \\
& =\sum_{n_{A}} c\left(n_{A}\right)\left|n_{A}\right\rangle
\end{aligned}
$$

where $c\left(n_{A}\right)=0$ if $n_{A}$ is odd, and equals $c\left(n_{A}\right)=$ $\left(\frac{\xi^{2}-1}{2\left(\xi^{2}+1\right)}\right)^{n_{A} / 2} \frac{\sqrt{n_{A} !}}{\left(n_{A} / 2\right) !}$ when $n_{A}$ is even, with $\xi^{2}=\alpha^{2}+1$.

The next step is generation of the cat state from the spin squeezed state by carrying out a QND measurement of $\hat{J}_{z}$. Towards this end we send off resonant right circular polarised light in a coherent state with the mean number of photons $N_{p}$ along the $z$ axis through the sample (the bottom level scheme in figure 1a). The temporal evolution of the light and spins is then given by $U=\exp \left(-i a \sum_{i}|\downarrow\rangle_{i}\langle\downarrow| \hat{a}_{R}^{\dagger} \hat{a}_{R}\right)$. We define a translated creation operator by $\hat{a}_{R}=\sqrt{N_{p}}+\hat{a}_{R}^{\prime}$ and the translated quadrature operators $\hat{x}_{R}=\left(\hat{a}_{R}^{\prime}+\hat{a}_{R}^{\prime \dagger}\right) / \sqrt{2}$, $\hat{p}_{R}=-i\left(\hat{a}_{R}^{\prime}-\hat{a}_{R}^{\prime \dagger}\right) / \sqrt{2}$ so that the unitary evolution takes the form $U=\exp \left[-i a \hat{n}_{A}\left(N_{p}+\sqrt{2 N_{p}} \hat{x}_{R}\right)\right]$ where we have dropped a term proportional to $\hat{a}_{R}^{\prime \dagger} \hat{a}_{R}^{\prime}$. The term proportional to $N_{p}$ does not modify the state of the light, but it rotates the atomic spin. This term can be taken into account at later stages of the protocol and is omitted from now on. The part of the unitary transformation relevant for the QND measurement is therefore $U=\exp \left[-i \beta \hat{n}_{A} \hat{x}_{R}\right]$ where $\beta=a \sqrt{2 N_{p}}$.

Initially light is in the coherent state $\psi_{R}=$ $\int d x_{R} \exp \left[-x_{R}^{2} / 2\right]\left|x_{R}\right\rangle$ and the spin squeezed state of atoms is given by eq. (44). After the interaction of light and atoms, one measures the $p_{R}$ quadrature and finds an outcome $p_{R}$. The state of the atoms conditional on this outcome is

$$
\psi_{A}\left(p_{R}\right)=\sum_{n_{A}} c\left(n_{A}\right) \exp \left[-\left(\beta n_{A}-p_{R}\right)^{2} / 2\right]\left|n_{A}\right\rangle .
$$

For suitable values of $\alpha, \beta, p_{R}$, this is a cat state. In order to show this one should express the state in the $x_{A}$ and $p_{A}$ basis. This can be done using the matrix elements $\left\langle p_{A} \mid n_{A}\right\rangle=$ $\pi^{-1 / 4}\left(2^{n_{A}} n_{A} !\right)^{-1 / 2} H_{n_{A}}\left(p_{A}\right) \exp \left[-p_{A}^{2} / 2\right]$ where $H_{j_{z}}\left(j_{y}\right)$ are the Hermite polynomials. A numerical calculation of $\psi_{A}\left(p_{R}\right)$ in the $p_{A}$ basis is given in figure 2 (solid line). To facilitate the analysis of the obtained state we derive approximate analytical expressions for $\psi_{A}\left(p_{R}\right)$ in the 
$x_{A}$ and $p_{A}$ basis. First note that using the Stirling expansion, the coefficients $c\left(n_{A}\right)$ can be approximated by $c\left(n_{A}\right) \simeq\left(\frac{\xi^{2}-1}{\xi^{2}+1}\right)^{n_{A} / 2}$ when $n_{A}$ is even. Inserting this expression into eq. (5) we find that

$$
\begin{gathered}
\psi_{A}\left(p_{R}\right) \simeq \sum_{n_{A} \text { even }} \exp \left[-\beta^{2}\left(n_{A}-\mu\left(p_{R}\right)\right)^{2} / 2\right]\left|n_{A}\right\rangle(6) \\
\text { where } \mu\left(p_{R}\right)=\frac{p_{R}}{\beta}+\frac{1}{2 \beta^{2}} \ln \left(\frac{\xi^{2}-1}{\xi^{2}+1}\right) \simeq \frac{p_{R}}{\beta} . \quad \text { (7) }
\end{gathered}
$$

The distribution of number states is thus a Gaussian with the mean $\mu\left(p_{R}\right)$ and the variance $1 / \beta$. We now use the fact that the initial state is strongly squeezed in $x_{A}$, see eq. (44). Thus we can use the approximation $n_{A} \simeq p_{A}^{2} / 2$ in eq. (6). This implies that the wave function in $p_{A}$ representation is centered around the two values $\pm \sqrt{2 \mu\left(p_{R}\right)}$ with the variance $1 /\left(\sqrt{2 \mu\left(p_{R}\right)} \beta\right)$. Hence the wave function is

$$
\begin{aligned}
\left|\psi\left(p_{R}\right)\right\rangle= & \int d p_{A}\left(\exp \left[-\left(p_{A}-\sqrt{2 \mu}\right)^{2} \beta^{2} \mu\right]\right. \\
& \left.+\exp \left[-\left(p_{A}+\sqrt{2 \mu}\right)^{2} \beta^{2} \mu\right]\right)\left|p_{A}\right\rangle \\
= & \int d x_{A} \exp \left[-\frac{x_{A}^{2}}{4 \beta^{2} \mu}\right] \cos \left[x_{A} \sqrt{2 \mu}\right]\left|x_{A}\right\rangle
\end{aligned}
$$

where the second equality if obtained from the first by taking the Fourier transform, and the notations are simplified as $\mu=\mu\left(p_{R}\right)$. Note that the Hermite polynomials with even index are even functions of $p_{A}$ which explains the + sign between the two terms in eq. (8). As seen in figure 2, eq. (8) is a good approximation to the exact wave function (dotted line).

To verify that the cat state has indeed been obtained, the measurements of spin components $\hat{x}_{A}$ and $\hat{p}_{A}$ should be carried out. The results of the $\hat{p}_{A}$ measurement should be distributed according to the 2 broad peaks described by eq. (8). The condition for observation of two distinct peaks is that the width of each of them should be less than the distance between them. The $\hat{x}_{A}$ measurement should exhibit an interference pattern due to the cosine in eq. (91). This interference pattern is the proof that the two peaks found in the $\hat{p}_{A}$ measurement are coherent, and therefore that a quantum superposition between atomic spins pointing in two different directions has been created. The condition for observation the interference is that the period of the cosine should be less than the width of the Gaussian in (9). In fact both conditions lead to the same inequality, $\mu \geq 1 / \beta$. This condition means that by the QND measurement of the population one has removed the components with small $n_{A}$, i.e. the components near the origin $p_{A}=0$, from the squeezed state leaving a state with two peaks.

There is a second condition which is that the average population $\mu$ is limited by the degree of spin squeezing obtained in the first step of our protocol: $\mu \leq \xi^{2}$. This condition has two origins. The first is that the probability of finding values of $\mu$ which exceed $\xi^{2}$ is exponentially small. The second is that when this condition is violated it becomes impossible to measure the interference pattern in $x_{A}$ basis, and thereby to prove that the state so obtained is indeed a coherent superposition.

Putting these two conditions together we obtain the condition $\beta \xi^{2}>1$ which relates the precision $1 / \xi$ with which one can measure the $\hat{x}_{A}$ quadrature to the precision $1 / \beta$ with which one can measure the number operator $\hat{n}_{A}$. Using the expressions for $\beta$ and $\xi$ in terms of $a$, we can reexpress this condition as

$$
\xi^{2} \geq N_{a}^{1 / 3}
$$

Thus a superposition state can be produced if a high degree of squeezing can be realised with a small number of atoms. In what preceeds we have assumed that the quantum states are pure and that there is no noise present. Below we consider decoherence of the atomic state caused by the non-ideal QND measurements and describe a possible experimental realization of the proposal.

The off resonant probe light used for the QND measurements will cause (weak) real excitations of atoms leading to an incoherent spontaneous process [12]. The significance of this process can be assessed in terms of accessible experimental parameters. A first key parameter is the resonant optical depth of the atomic sample, $\kappa_{0}=\sigma N_{a} / A$ where $\sigma$ is the cross section. The forward scattering amplitude of light is proportional to $\frac{\gamma}{2} /(i \Delta+\gamma / 2)$ where $\gamma$ is the linewidth and $\Delta$ is the detuning with respect to resonance. Hence the optical depth at detuning $\Delta \gg \gamma$ is $\kappa_{\Delta}=\kappa_{0} \gamma^{2} / 4 \Delta^{2}$ and the phase shift (or polarisation rotation) of light traversing the sample is $\theta_{\Delta}=\kappa_{0} \gamma / 2 \Delta$. The parameter $a$ defined in eq. (3) is the phase shift or polarisation rotation per atom, hence equal to $a=\theta_{\Delta} / N_{a}$. The degree of squeezing that can be obtained is $\xi=a \sqrt{N_{a} N_{p}} / 2$. Decoherence caused by the probe light can be described by the degree of optical depumping, $\eta=\kappa_{\Delta} N_{p} / N_{a}$ which can be interpreted as the probability that an atom makes an incoherent transition. All these parameters can be put together in the equation $\xi^{2}=\kappa_{0} \eta / 4$ relating the degree of squeezing, the resonant optical depth and the degree of optical depumping. The degree of optical depumping must obey the condition $\eta \leq 1 / \xi^{2}$ otherwise the coherence of the squeezed state or the superposition state will be destroyed. This condition follows from the fact that a fraction $1 / \xi^{2}$ of the spins are pointing in the $-z$ direction and the other spins are pointing in the $+z$ direction. Hence on average $N_{A} / \xi^{2}$ atoms can decohere before the coherence of the superposition state, or of the parent squeezed state, is seriously affected. Thus the figure of merit for generation of squeezing and entanglement is the resonant optical depth of the sample which limits the degree of spin squeezing to $\xi^{2} \leq \frac{1}{2} \sqrt{\kappa_{0}}$. Using these conditions and eq. (10), one finds that the condition for realising a superposition state can be written as

$$
\kappa_{0} \geq 4 N_{a}^{2 / 3},
$$

that is a large optical depth must be achieved with a small number of atoms. This means that sending light 
through the sample along orthogonal directions for the two steps of the protocol is not optimal. Rather it seems more practical to confine the atoms to a cylinder of area $A$ and length $l$, with light sent along the long axis of the cylinder, and to rotate the atomic spins between successive measurements.

The condition eq. (11) can be achieved in, e.g., an alkali metal BEC. Atomic density of the order of $10^{15} \mathrm{~cm}^{-3}$ for $4 \cdot 10^{5}$ atoms has been reported in 14] for a cigar shaped condensate with the optical density along the long axis on the order of $10^{4}$. This is marginally close to the condition eq. (11). The degree of squeezing required for cat state generation under the above conditions is $\xi^{2} \approx 50$. An additional improvement in the efficiency of the spin measurement of a small number of atoms can be achieved by placing the atomic sample in a low-finesse symmetric standing wave cavity with the mirror transmission coefficients $T=1-R$. If the single pass absorption probability and the single pass phase rotation angle $\theta$ are much smaller than $T$, then the phase shift/polarisation rotation of light exiting the cavity is $\theta^{c}=2 \theta / T$ and the degree of optical depumping becomes $\eta^{c}=2 \eta / T$. Thus the effect of the cavity can be summarized by introducing an effective cross section $\sigma^{c}=2 \sigma / T$. The condition eq. (11) for realisation of a mesoscopic superposition state therefore becomes $2 \kappa_{0} / T>4 N_{a}^{2 / 3}$. For a BEC sample with the density of $10^{15} \mathrm{~cm}^{-3}$ placed in a low finess cavity with $T=5 \%$, this condition is met with $10^{3}$ atoms, which according to eq. (10) requires a feasible degree of spin squeezing $\xi^{2} \approx 10$ for generation of the superposition state. Note that the life time of the coherent superposition state is close to the life time of the parent spin squeezed state, $\tau_{c} \xi^{-2}$ [15], where $\tau_{c}$ is the coherence time of the atomic ground state, which for a BEC is at least $100 \mathrm{~m}$ sec 16].

Finally let us mention some additional experimental conditions. We have assumed above that the initial atomic spin state is perfectly polarized. In practice the degree of spin polarisation obtained by optical pumping in a vapor can reach $99 \%$ [9], which should be sufficient to generate squeezing up to approximately $\xi^{2}=100$. The degree of spin polarization in a BEC can be even higher because only certain magnetic states can be trapped. Another challenge associated with the cylindrical configuration is that the atomic spins must be very precisely rotated between each QND measurement (using either optical or radio frequency pulses). It is easy to check that the precision of this rotation must satisfy $\Delta \theta<1 /\left(\xi^{2} \sqrt{N_{a}}\right)$ which for the free space scenario with a BEC corresponds to the precision $\Delta \theta \simeq 3 \times 10^{-5}$. For the low finesse cavity scenario a lower precision of $\Delta \theta=1 / 300$ is necessary.

In summary we have proposed a method for generating a coherent superposition of states in which the collective spin of an atomic ensemble points in two slightly different directions. It appears to be realisable using present technology by combining the methods used for QND measurements of collective atomic variables with a BEC in a bad cavity. Preliminary experimental work towards realisation of this proposal is under way. This mesoscopic superposition state uses the ground state atomic spins which means that it is relatively long lived. It can therefore in principle be used as an offline non deterministic resource for quantum logic as described in [2]. We will report on this in a future publication and in particular we will show that the methods described here can be used for purifying the entanglement between two distant atomic ensembles.

We acknowledge financial support from the Danish National Research Foundation, from the Communauté Française de Belgique under grant ARC 00/05-251, from the IUAP programme of the Belgian government under grant $\mathrm{V}-18$, from the EU through projects EQUIP, RESQ, QUICOV and CAUAC.
[1] E. Schrödinger, Die Naturwissenschaften 23 (1935) 807

[2] T. C. Ralph et al, quant-ph/0110115

[3] J.M. Raimond et al, Phys. Rev. Lett., 79, 1964 (1997)

[4] C. H. van der Wal et al, Science 290, 773 (2000); J. R. Friedman et al, Nature 406, 43 (2000)

[5] J. I Cirac et al, Phys. Rev. A 57, 1208 (1998); J. Ruostekoski et al, Phys. Rev. A 57, 511 (1998)

[6] S. Song et al, Phys. Rev. A 415261 (1990); B. Yurke et al, Phys. Rev. A 421703 (1990)

[7] H.J. Kimble, Physica Scripta T76, 127 (1998); G.R. Guthohrlein et al, Nature, 414, 49 (2001); G. Rempe, Physics World13, 37, (2000)

[8] A. Kuzmich and E. S. Polzik, Phys. Rev. Lett. 85, 5639 (2000)
[9] B. Julsgaard et al, Nature 413, 400 (2001)

[10] J. Hald et al, Phys. Rev. Lett. 83, 1319 (1999)

[11] A. Kuzmich et al, Phys. Rev. Lett. 85, 1594 (2000)

[12] L. M. Duan et al, Phys. Rev. Lett. 85, 5643 (2000)

[13] C. Schori et al, Phys. Rev. Lett. 89, 057903 (2002); A. Kuzmich and E.S.Polzik, to appear in "Quantum Information with Continuous Variables". Ed. S. Braunstein, Kluwer.

[14] H. Ott et al, Phys. Rev. Lett. 87, 230401 (2001) and C. Zimmerman, private communication.

[15] S. F. Huelga et al, Phys. Rev. Lett., 79, 3865 (1997)

[16] D. S. Hall et al, Phys. Rev. Lett., 81, 1543 (1998) 


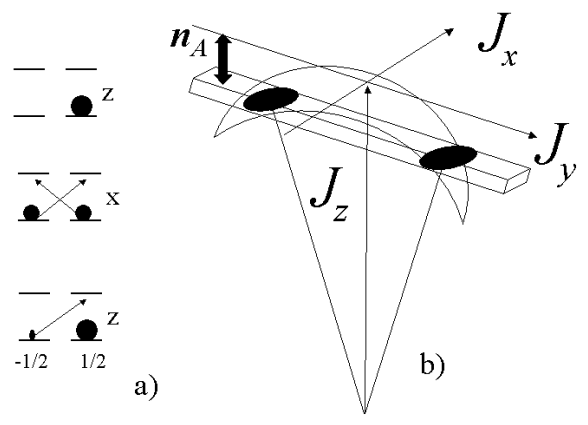

FIG. 1: Generation of the superposition spin state. a) Atomic levels and interaction with light. Both ground and excited states are spin $1 / 2$ states. The top diagram shows the initial coherent spin state in $z$ representation. The medium diagram shows the same state in $x$ representation along with the virtual transitions used for generation of the spin squeezed state. The bottom diagram shows the spin squeezed state in $z$ representation along with the interaction used for the cat state generation. b) The spin squeezed state generated by a QND measurement using the first pulse of light is represented by the "banana" shape. The following QND measurement of the number operator $\hat{n}_{A}$ corresponds to intersecting the "banana" with a plane of a finite width. Thus the final state has two components lying at positive and negative values of $J_{y}$, i.e. it is a superposition state.

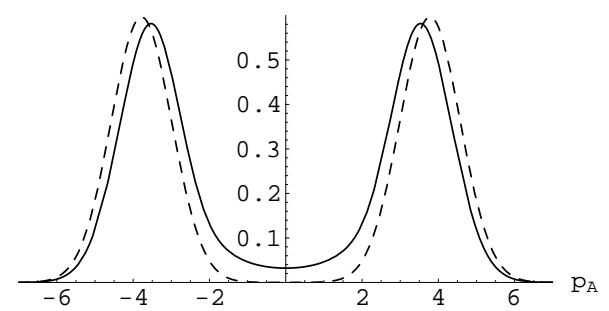

FIG. 2: The solid line shows the amplitude of the state $\psi_{A}\left(p_{R}\right)$, eq. (5), in the $p_{A}$ basis for parameters $\xi^{2}=20$, $p_{R} / \beta=7, \beta=1 / 3$. The dotted line shows the approximation eq. (8) for the same values of the parameters. 